\title{
AVALIAÇAOO DA ATIVIDADE TERAPEUTICA DO ALBENDAZOL EM RATOS EXPERIMENTALMENTE INFECTADOS COM STRONGYLOIDES VENEZUELENSIS
}

\author{
Vicente AMATO NETO (1), Pedro Luiz Slya PINTO (2), Antonio Augusto Baillot MOREIRA (2), \\ Rubens CaMPOS (2). Eunice José de SANT'ANa (2), Eduardo Virgllio LEVAI (2), Lilliana Apareciđa \\ Araújo PADILHA (2) e Clovis Kiomitsu TAKIGUTI (3)
}

\section{R E S U M O}

Com a finalidade de demartı́r mais precisamente o espectro de açăo do albendazol, foi estudada a atividade terapêutica desse anti-helmintico em ratos experimentalmente infectados com Strongyloides venezuelensis, tendo sido usada, como termo de comparação, a ação do cambendazol e do mebendazol, dois outros benzimidazólicos. Os três compostos mostraram-se eficientes quando utilizadas doses únicas de $6,75,12,5,25$ e $50 \mathrm{mg} / \mathrm{kg}$, pois motivaram desaparecimento total das formas adultas no intesiinu. Com a posologia de $5 \mathrm{mg} / \mathrm{kg}$ sucederam porcentagens médias de reduçếs dos números de vermes de $87 \%, 98 \%$ e $80 \%$, respectivamente, como decorrência do emprego do albendazol, do cambendazol e do mebendazol, traduzindo superioridade da segunda droga citada.

UNITERMOS: Estrongiloidíase - Strongyloides venezuelensis - Infecção experimental em ratos - Tratamento - Albendazol

\section{N T ROD U G A O}

A partir de 1980 o albendazol (metil 5-propiltiobenzinidazol-2-carbamato) passa a ser considerado perspectiva promissora na terapêutica de helmintíases humanas $13,14,17,18,19$.

Observações realizadas para verificar a eficácia desse composto e demarcar precisamente o aspectro de ação a ele pertinente, permitiram apreciaçāo satisfatória e conclusiva relativamente à ancilostomíase, à escaridíase, à enterobíase e à tricocefalíase $2,3,5,6,8,9,10,12,16$. Todavia, as opiniōes sobre a estrongiloidiase foram discordantes, de molde a não permitir posição definitiva, como mormente os informes de AMATO NETO \& col. ${ }^{4}$ e de ROSSIGNOL \& MAISONNEUVE 20 evidenciaram, marcando clara contra. dição, a despeito da adoção de uma mesma conduta. Daí a validade do objetivo desta investigação que, experimentalmente, por meio de modelo calcado na infecção de ratos pelo Strongyloides venezuelensis (BRUMPT, 1984) procura situar os atributos da substância em questão, confrontada com outras, ou seja, o cambendazol e o mebendazol, efetivas contra a estrongiloidiase murina ${ }^{15}$.

\section{MATERIAL E METODOS}

Utilizamos ratos albinos com peso corporal médio de $150 \mathrm{~g}$, procedentes do Biotério Central da Faculdade de Medicina da Universidade de São Paulo.

Hospital das Clinicas, da Faculdade de Medicina da Universidade de São Paulo. Laboratório de Invastigação Múdica - Parasitologia, São Paulo, Brasil.

(1) Chefe do Laboratório de Investigação Médica - Parasitologia

(2) Membro do Laboratório de Investigação Médica - Parasitologia

(3) Professor-livre-docente do Departamento de Medicina Preventiva, da Faculdade de Medicina da Universidade de São Paulo, São Paulo, Brasil 
AMATO NETO, V.; PINTJ, D. L. S.; MORFira, A. A. B.; CAMPOS, R.; SANT'ANA, E. J. de; LEVAr, E. V.; PADILHA, L. A. A. \& TAKIGUTI, C. K. - Avaliação da atividado terapêutica do albendazol em ratos experimentalmente infectados com Strongyloides venexuelensis. Rev. Inst. Med. trop. São Paulo, 28:181-184, 1986.

Isolamos a cepa de $\mathbf{S}$. venezuelensis a partir de Rattus norvegicus, capturados pelo Departamento de Controle de Zoonoses e Vigilância Sanitária, da Prefeitura do Município de São Paulo. A propósito, adotamos a metodologia indicada por BRENER \& CHAIA $^{7}$ para obtenção do $S$. ratti.

Usamos os seguintes antiparasitários: albendazol (comprimido com $400 \mathrm{mg}$; lote exp. 08; "Searle do Brasil S/A"; mebendazol (sal puro, cedido por G. Chaia); cambendazol (suspensão com $6 \mathrm{mg} / \mathrm{ml}$; lote 7 170; adquirido ho comércio - "Cambem, Ceil - Divisão Farmacêutica Lafi"). Juntamos água aos dois primeiros e, à preparação resultante adicionamos pequena quantidade de goma arábica em pó, para facilitar a homogeneidade.

Dividimos os animais em grupos de três e inoculamos em todos, pela via subcutânea, $\mathbf{5 0 0}$ larvas filarióides de $\mathbf{S}$. venezuelensis.

Os esquemas corresponderam a $5,6,75,12,5$, 25 e $50 \mathrm{mg} / \mathrm{kg}$ no que tange aos três anti-helmínticos, administrados oralmente, em dose única, no oitavo dia após a infecção. Como controle, mantivemos ratos năo tratados.

A avaliação da eficácia teve base na pesquisa das fêmeas partenogenéticas na parede intestinal. Para isso, sacrificamos os roedores no décimo-segundo dia depois da infecção e retiramos os quinze centímetros iniciais do intestino delgado. Com o auxílio de fio amarrado em uma das extremidades e de bastáo fino de madeira exteriorizamos a mucosa intestinal. Colocamos o fragmento invertido em um tubo de ensaio (25 × $16 \mathrm{~cm}$ ) com água a $42^{\circ} \mathrm{C}$ e, decorrida uma hora, centrifugamos o líquiido e examinamos o sedimento por meio de microscópio entomológico, contando o número de vermes, quando presentes. A média de fêmeas recuperadas nos grupos de animais tratados com cada um dos diferentes medicamentos foi processada estatisticamente, pelo teste de neumanKeuls, através de análise de variância, com estudos dos contrastes 22 .

\section{RESULTADOS}

Os resultados atinentes às três drogas indicaram comportamentos similares, se consideradas as doses de $6,75,12,5,25$ e $50 \mathrm{mg} / \mathrm{kg}$, que promoveram desaparecimento total das formas adultas. Por seu turno, a quantidade de $5 \mathrm{mg} /$ kg permitiu verificar atividades de $87 \%, 98 \%$ e $80 \%$ do albendazol, do cambendazol e do mebendazol, respectivamente, traduzindo essas taxas reduções médias dos números de fêmeas partenogenéticas recolhidas dos animais tratados, servindo como comparação o valor congênere correspondente às recuperadas no grupo controle e, precisamente, estipulado como 33, tido então como $100 \%$ para fins de cotejo. Essas constataçōes encontram-se assinaladas nos Quadros I e II e o que comprovamos com a posologia por último comentada, para cada um dos três compostos, ensejou a análise estatística proposta, com necessidade, previamente ao teste, de efetuar a transformação $x^{\prime}=\sqrt{\mathbf{X}+0,5}$, sendo $X$ ' o valor da variável transformada e $X \circ$ da original. $A$ análise de variância revelou que as médias de fêmeas recuperadas, quando consideradas duas a duas, não são iguais $\left(F_{3,8}=37,28\right)$. O teste de intervalo múltiplo indicou que os contrastes controlecambendazol, controle-albendazol, controle-mebendazol, mebendazol-cambendazol e albendazol-cambendazol são estatisticamente significan. tes ao nível de $5 \%$, enquanto que o mebendazol-albendazol mostrou que as médias sạo iguais ao mesmo nivel de $5 \% 22$.

\section{Q U A D R O I}

Atividade de três medicamentos anti-helmínticos na infeçäo experimental de ratos pelo Strongyloides venezuelensis: porcentagem de reduçāo do número de vermes recuperados no intestino apos tratamento

\begin{tabular}{rccc}
\hline \multirow{3}{*}{$\begin{array}{c}\text { Dose única } \\
(\mathrm{mg} / \mathrm{kg})\end{array}$} & Albendazol & Cambendazol & Mebendazol \\
\cline { 2 - 4 } & $\mathbf{3 7}$ & 98 & 80 \\
$\mathbf{5}$ & 100 & 100 & 100 \\
$\mathbf{6 , 7 5}$ & 100 & 100 & 100 \\
12,5 & 100 & 100 & 100 \\
25,5 & 100 & 100 & 100 \\
50 & & & \\
\hline
\end{tabular}

\section{DISGUSSAO}

A despeito das reconhecidas limitações das tentativas de extrapolar dados advindos de análises experimentais, acerca o valor terapêutico de drogas, a disponibilidade de modelo para a estrongiloidíase tem contribuído, nesse campo, de maneira eficiente e econômica, para registrar deduçōes aproveitáveis 11,15. Em espe- 
AMATO NETO, v.; PINTO, P. L. S.: MOREIRA, A. A. B; CAMPOS, R.; SANT'ANA, E. J. de; LEVAI, E. V.; PADILHA,

L. A. A. \& TAKIGUTI, C. K. - Avaliação da atividade terapêutica do albendazol em ratos experimentalmente infectados com Strongyloides venezuelensis. Rev. Inst. Med. trop. São Paulo, 28:181-184, 1986.

Q U A D R O II

Atividade de três medicamentos anti-helmínticos na infeç̧āo experimental de ratos pelo Strongyloides venezuelensis: número médio de vermes recuperados no intestino apos uso de dase única de $5 \mathrm{mg} / \mathrm{kg}$, em comparação com o sucedido em animais não tratados

\begin{tabular}{|c|c|c|}
\hline Medicamento & $\begin{array}{l}\text { Número médio e } \\
\text { porcentagem de } \\
\text { vermes recupera- } \\
\text { clos }\end{array}$ & $\begin{array}{l}\text { Porcentagem de } \\
\text { redução do nú- } \\
\text { mero de vermes } \\
\text { recuperados }\end{array}$ \\
\hline Albendazol & $4,3 \quad(13)$ & 87 \\
\hline Combendazol & $0,0 \quad(2)$ & 98 \\
\hline Mebendazol & $6,6 \quad(20)$ & 80 \\
\hline Nāo tratados & $33 \quad(100)$ & - \\
\hline
\end{tabular}

Três animais constituiram cada um dos quatro grupos

cial quanto ao albendazol e sua posição frente à verminose humana em apreço, os fatos até agora enunciados geraram contrađiçōes provavelmente por terem os pesquisadores recorrido a metodologias diferentes. Essa circunstância e a ausência de especulações que delimitem os atributos desse remédio em confronto com outros benzimidazólicos já avaliados em hospedeiro murino, justificaram a presente mensuração da capacidade dele, em paralelo com a do cambendazol e do mebendazol.

As deduções que apresentamos referem-se à ação dos compostos na parasitose em fase patente, uma vez que a infecção pelo $\mathbf{S}$. venezuelensis tem -periodo pré-patente de aproximadamente quatro dias e a administração deles teve luoar no oitavo dia 2k. Assim, foi-nos possivel comprovar que com dosagens superiores a 5 $\mathrm{mg} / \mathrm{kg}$ houve desempenho semelhante das três substâncias, evidenciado pelo desaparecimento total das fêmeas partenogenéticas na parede intestinal. Contudo. quando escolhida a quantidade de $5 \mathrm{mg} / \mathrm{kg}$ ficou evidente que o cambendazol mostrou-se superior, situando-se o albendazol e o mebendazol em posição menos proeminente, consoante o processamento estatístico e o que a terapêtica clínica já comprovou 1. Valorizadas devidas ressalvas, $e$ lícito insinuar que tais constataçōes possam ser reportadas para a estrongiloidíase humana.

Debelar a verminose em foco é intento hoje bastante relevante, uma vez que ela, ao lado de outros percalços, gera comprometimentos orgânicos graves, claramente evidenciados na doença disseminada, manifesta como fruto da influência de vários fatores e, outrossim, de imunodepressão de diferentes naturezas, entre as quais situam-se a medicamentosa, a instituida após transplante de órgãos e a da sínđrome da imunodeficiência adquirida (AIDS). Considerada essa ponderação, pode-se enfatizar devidamente o propósito maior da pesquisa que relatamos.

\section{SUMMARY}

Evaluation of the activity of albendazole in the therapy of experimentally-infected rats

In order to settle the precise spectrum of activity of albendazole, its efficacy was tested in the treatment of Strongyloides venezuelensis in experimentally-infected rats. The results were compared to those obtained with two other benzimidaloze derivatives, cambendazole and mebendazole. The three compounds were shown to be effective in eradicating intestinal adult worms completely, when single doses of $6.25,12.5,25$ of $50 \mathrm{mg} / \mathrm{kg}$ were used. However when smaller doses were employed $(5 \mathrm{mg} / \mathrm{kg}$ ) worm burdens were reduced in $87 \%, 98 \%$ and $80 \%$ by albendazole, cambendazole and meben. dazole respectively.

\section{REFERENGIAS BIBLIOGRAFIGAS}

1. AMATO NETO. V.; SINTO, T.; PEDRO, R. J.: LEVI, G .C.; TSUKUMO, M. K. K.: MORAES, v. M. C. \& CORRea, L. L. - Nossas observacöes iniclais sobre a aficácia do cambendazole no tratamento $d a$ estrongiloídíase. Rev. Inst. Med. trop. S. Paulo, 20: 161-163, 1978.

2. AMATO NETO, V.; MOREIRA, A. A. B.; CAMPOS, R.; LAZZARO, E. S. M.; CHIARAMELLI, M. C. G.; CAS TILHO, V. น. P.; GOMES, A. E. C. \& PINTO, P. L. S. - Tratamento da ancilostomíase por meio do albendazol. Rev. Inst. Med. trop. S. Paulo, 25: 42-46, 1983.

3. AMATO NETO, V.; MOREIRA, A. A. B.; CAMPOS, R.: LAZZARO, E. S. M.: CHIARAMELLI, M. C. G.; PINTO, P. L. S.; NISHIOKA, S. A.; LEITE, R. M. \& SILVA, G. R. - Tratamento da ancilostomíase, ascaridíase e tricocefalíase por meio do albendazol ou do mebendazol. Rev, Inst. Med. trop. S. Paulo, 25: 294-299, 1983.

4. AMATO NETO, V.; MOREIRA, A. A. B.; CHIARAMELII, M. C. G.; LEME, J. M. T. P.; CHIARAMELLI, D. R.; CAMPOS, R.; PINTO, R. L. S.; SANT'ANA, E. J. \& ROCHA, S. - Demarcaçăo da atividade anti-helmintica do albendazol: estudo referente à estrongiloidiase hu. mana. Rev. Inst. Med. trop. S. Paulo, 27: 95-98, 1985 
AMATO NETO, V.; PINTO, P. L. S.; MOREIRA, A. A. B.; CAMPOS, R.; SANT'ANA, E. J. de; LEVAI, E. V.; PADILHA, L. A. A. \& TAKIGUTI, C. K. - Avaliaçăo da atividade terapêutica do albendazol em ratcs experimentalmente inrectados com Strongyloldes venezuelensis. Fev. Inst. Med. trop. Säo Paulo, 28:181-184, 1986.

5. AMATO NETO, V.; CASTILHO, v. L. P.; MOREIRA, A. A. B.; SANT'ANA, E. J.; PINTO, P. L. S.; CAMPOS, R. \& PADILELA, L. A. A. - Eficácia do albendazol no tratamento da enterobíase. Rev. Inst. Med. trop. S. Paulo, 27: 143-144, 1985.

6. BARANSKI, M. C.: SILVA, A. F. \& GUIMARAES, L. M. - Tratamento das helmintiases intestinais pelo albendazole, novo antithelmíntico do grupo dos benzimidazóis. Estudo duplo cego. In: CONGRESSO DA SOCTEDADE BRASILEIRA DE MEDICINA TROPICAL, 18., Ribeiráo Preto, 1982. Programa e resumos. Ribeirão Preto, Gráfica São Francisco, 982. p. E19.

7. BRENER, Z. \& CHAIA, G. - Isolamento e manutençầ do "Strongyloides ratti" (Sandground, 1925) em condiçōes de laboratório. Rev, bras. Biol., 20: 447-451, 1960.

8. CAMILlo-CoURA, L.; SOLI, A. S. V. \& WILLCOX, H. P. F. - Ensaio com o albendazole no tratamento das helmintfases intestinais em crianças. In: CONGRESSO DA SOCIEDADE BRASILEIRA DE MEDICINA TROPICAL, 19., Rio de Janeiro, 1983. Programa e resumos, Rio de Janeiro, Imprinta, 1983, res. n.o 257 , p. 114 .

9. CAMILLO-COURA, L.; SOLI, A. S. V.; IIMA, N. S.; PEIXOTO, T. C. \& WILLCOX, H. P. F. - Tratamento das helmintiases intestinais com albendazole. Estudo duplo-cego. In: CONGRESSO DA SOCIEDADE BRASILEIRA DE MEDICINA TROPICAL, 19, Rio de Ja neiro, 1983: Programa e resumos. Rio de Janeiro, Imprinta, 1983. res. n.० 258, p. 114.

10. CAMPOS, R.; MOREIRA, A. A. B.; CASTILHO, V. L. P.; AMATO NETO, V.; GUIZEHINI, E. \& PINTO, P. L. S. - Tratamento da ascaridiase e da tricocefaliase por meio do albendazol. Arch. bras. Med., 57: 185-186, 1983

11. ChAlA, G. - Atividade do 2-(4'tiazolil)-benzimidazol "Tiabendazol" em ratos experimentalmente infectados pelo Strongyloides ratti. Rev. Inst. Med. trop. S. Paulo, 5: 154-159, 1963.

12. FERNANDES, P.; NASCIMENTO, J. L.; BARROSO JUNIOR, G. \& SILVA, F. J. - Albendazole, nova conquista no tratamento das polihelmintiases. In: CONGRESSO DA SOCIEDADE BRASILEIRA DE ME. DICINA TROPICAL, 18., Ribeiräo Preto, 1982. Pro. grama e resumos. Ribeirăo Preto, Gráfica São Francisco, 1982. p. El4.

13. GARIN, J. P.; MOJON, M.; PIENS, M. A. \& ROSSYGNOL, J. F. - Albendazole efficacy on a long term follow up in patients with intestinal helminthlasis.
(Apresentado no 10th International Congress on Tropical Medicine and Malaris, Manilha, Filipinas, 1980).

14. GARIN, J. P.; MOJON, M.; PIENS, M. A. \& ROSSIGNOL, J. F. - Albendazole in the treatment of in testinal helminthiasis in humans: an Euro-African double blind multicenter trial. (Apresentado no 10th International Congress on Tropical Medicine and Malaria, Manilha, Filipinas, 1980)

15. GROVE, D. I. - Strongyloides ratti and $\mathbf{S}$. stereoralis: the effects of thiabendazole, mebendazole, and cambendazole in infected mice. Amer. J. trop. Med. Hyg., 31: 469.476, 1982,

16. MARCONDES, J.; MEIRA, D. A.; GUSHIKEN, T. \& VILLAÇA, E. C. - Tratamento das helmintíases intestinais com albendazol em individuos habitantes do $\mathrm{Mu}$ nicipio de Humaitá, Estado do Amazonas. In: CONGRESSO DA SOCIEDADE BRASILEIRA DE MEDICINA TROPICAL. 19., Rio de Janeiro, 1983. Programa e resumos. Rio de Janeiro, Imprinta, 1983. res. n.a 265, p. 117-118.

17. PENE, P. - The albendazole in treatment of intestinal parasitoses. (Apresentado no 10th International Congress on Tropical Medicine and Malaria, Manilha, Filipinas, 1980).

18. PENE, P.; MOJON, M,; GARIN, J. P.; COULAUD, J. P. \& ROSSIGNOL, J. F. - Albendazole: a new broad spectrum anthelmintic. Double-blind multicenter clinical trial. Amer. J. trop. Med. Hyg., 31: 263-266, 1982

19. RODRIGUES, L. D.; MARTIRANI, I. \& BRANDAO, J A. - Experimentação clínica com o albendazol, novo antihelmintico do grupo dos bensimidazólicos. In: CONGRESSO DA SOCIEDADE BRASILEIRA DE ME DICINA TROPICAL, 16., Natal, 1980. Resumos. NataI, 1980. res. n.० 85 .

20. ROSSIGNOL, J. F. \& MAISONNEUVE, F. - Albendazole: placebo-controlled study in 870 patients with intestinal helminthiasis. Trans. roy. Soc. trop. Med. HYrg., 77: 707-711, 1983

21. WERTHEIM, G. - Growth and development of Strongyloldes venezuelensis Brumpt, 1934 in the abino rat. Parasitology, 61: 381-388, 1970 .

22. ZAR, J. H. - Statistical Analysis. London, PrenticeHall, 1974. p. 151-155.

Recebldo para publicação em 26/6/1985 